\title{
Isolamento de fungos filamentosos em água utilizada em uma unidade de hemodiálise
}

\author{
Isolation of filamentous fungi from water used \\ in a hemodialysis unit
}

\author{
Samuel Dutra Varo ${ }^{1}$, Carlos Henrique Gomes Martins ${ }^{1}$, Miguel Jorge de Oliveira Cardoso ${ }^{1}$, \\ Flávio Garcia Sartori' ${ }^{1}$, Lílian Bueno Montanari ${ }^{1}$ e Regina Helena Pires-Gonçalves ${ }^{1}$
}

\begin{abstract}
RESUMO
A despeito da relativa freqüência de infecções fúngicas oportunísticas em pacientes sob hemodiálise, os reservatórios ambientais destes permanecem desconhecidos, embora alguns estudos recentes tenham correlacionado o suprimento de água como fonte desses microrganismos. O objetivo deste trabalho foi monitorar a qualidade micológica do sistema hídrico de uma Unidade de Hemodiálise, do interior do Estado de São Paulo, Brasil, no período entre abril e julbo de 2006. Foram coletadas amostras (15), de 1000mL em 7 pontos de distribuição de água empregando-se técnica da membrana filtrante $(0,45 \mu \mathrm{m})$. Foram isolados 116 fungos filamentosos, dos quais 47 (40,5\%) Trichoderma sp, 29 (25\%) Cladosporium sp, 16 (13,8\%) Aspergillus sp e 11 (9,5\%) Fusarium sp. Mediante os resultados, sugerimos que suprimentos de água para Unidades de Hemodiálise devam ser monitorados também quanto ao aspecto micológico, adotando-se medidas profiláticas eficazes que minimizem a exposição destes pacientes imunodeficientes a fontes aquáticas ambientais contaminadas.
\end{abstract}

Palavras-chaves: Água. Fluidos de hemodiálise. Qualidade microbiana da água. Hemodiálise. Fungos filamentosos.

\section{ABSTRACT}

Despite the relative frequency of opportunistic fungal infections among hemodialysis patients, the reservoirs for these microorganisms in the environment remain unknown, although some recent studies have made correlations with the water supply as their source. The objective of the present study was to monitor the mycological quality of the water system of a bemodialysis unit in the interior of the State of São Paulo, Brazil, over the period from April to July 2006. Fifteen samples of $1000 \mathrm{ml}$ were collected from seven water distribution points using the membrane filtration technique $(0.45 \mathrm{\mu m})$. A total of 116 filamentous fungus specimens were isolated, including 47 Trichoderma sp (40.5\%), 29 Cladosporium $s p$ (25\%), 16 Aspergillus $s p$ (13.8\%) and 11 Fusarium $s p$ (9.5\%). The results suggest that the water supply for hemodialysis units should also be monitored for mycological contamination, and that effective prophylactic measures should be adopted for minimizing the exposure of these immunodeficient patients to contaminated water sources in the environment.

Key-words: Water. Hemodialysis fluids. Microbial water quality. Hemodialysis. Filamentous fungi.

No Brasil, são estimados 50.000 pacientes renais crônicos distribuídos em 540 clínicas, sendo 45.000 em hemodiálise e 5.000 em diálise peritoneal ${ }^{43}$.

Doenças infecciosas são a segunda causa de morte em pacientes renais crônicos, superadas apenas pelas doenças cardiovasculares, sendo que septicemia ocorre em aproximadamente $75 \%$, destes pacientes ${ }^{1}$. Entre pacientes hemodialisados, baixos níveis de albumina plasmática, acesso vascular temporário e reuso de dialisadores são também fatores de risco associados ${ }^{42}$.
A patogênese das infecções associadas a pacientes hemodialisados deve-se principalmente a três fatores: relativo ao hospedeiro - insuficiente ativação das células T, deficiências tanto nas funções dos neutrófilos e macrófagos como na resposta humoral; relativo ao microrganismo - genes de virulência, propriedades de aderência e formação de biofilme; relativa à diálise - acesso vascular, rompimento da integridade da pele, prática de reuso de dialisadores e sistemas de tratamento de água ${ }^{102034}$.

A hemodiálise em pacientes portadores de insuficiência renal crônica (IRC) é um procedimento que manipula o sangue três

1.Laboratório de Pesquisa em Microbiologia Aplicada, Universidade de Franca, Franca, SP.

Endereço para correspondência: Dr. Carlos Henrique G. Martins. Av. Dr. Armando Salles Oliveira 201, 14404-600 Franca, SP.

Tel: 55 16 3711-8756; Fax: 55 16 3711-8873

e-mail: martinsc@unifran.br

Recebido em 3/11/2006

Aceito em 4/5/2007 
vezes por semana, durante cerca de 4 horas e num fluxo que varia entre 350 e $400 \mathrm{~mL} / \mathrm{min}$, sendo que apenas uma membrana semi-permeável separa o sangue do paciente do dialisado. A água utilizada para esse tipo de terapia deve apresentar qualidade em obediência ao recomendado pela legislação federal vigente ${ }^{33} \mathrm{e}$ deve sofrer constante vigilância para manter respeitados os níveis máximos de contaminantes, garantindo a saúde do paciente.

É usual a reutilização do capilar dialisador em um mesmo paciente, sendo necessário medidas como, enxágüe para retirada do sangue restante, seguido de limpeza química e de desinfecção. Reações em pacientes decorrentes de inadequada manutenção dos sistemas de água e reutilização dos capilares são descritas pela literatura científica ${ }^{26}$.

A incidência de infecções fúngicas, especialmente em hemodialisados, vem aumentando gradativamente ${ }^{1}$, sendo que, em geral, a colonização precede a doença ${ }^{7}$. A despeito desta relativa frequiência, o conhecimento da epidemiologia dessas infecções é pouco conhecido no Brasil, bem como os fatores associados e o impacto destas infecções em pacientes sobreviventes.

Embora o monitoramento microbiológico da água e do dialisado deva ser realizado no mínimo mensalmente ou imediatamente nos casos de reação pirogênica ou septicemia no paciente em diálise e, também, após modificações do tratamento de água ou sistema de distribuição ${ }^{15}$, no Brasil, assim como na legislação internacional, a análise bacteriológica de rotina da água usada nos procedimentos de hemodiálise não inclui a pesquisa e/ou identificação de fungos e, poucas são as publicações que têm relatado o encontro destes microrganismos nestas águas ${ }^{67}$.

Assim, neste estudo procurou-se monitorar qualitativa e quantitativamente estas águas como possível fonte de fungos filamentosos, potencialmente patogênicos, enfatizando a necessidade de implementação de normas quanto à análise micológica em águas utilizadas em unidades de hemodiálise.

\section{MATERIAL E MÉTODOS}

Amostragem. Este estudo foi submetido e aprovado pelo Comitê de Ética em Pesquisa (CEP) da Universidade de Franca.

As amostras de água foram coletadas em uma unidade de hemodiálise, vinculada a um hospital, localizada no interior do estado de São Paulo, no período de abril a julho de 2006, sendo obtidas de sete pontos específicos: 1) água de rede de abastecimento (adição de 2,0mL de tiossulfato de sódio a 1,8\% para neutralização de cloro residual); 2) do processo pósfiltração (pós-osmose); 3) do tanque, onde armazena-se toda a água filtrada, e de cada sala onde se realizam as sessões de hemodiálise, designadas como 4) Sala A; 5) Sala B; 6) Sala C; e 7) de máquinas previamente desinfetadas para reinício de sessões.

Todas as amostras (1 litro) foram colhidas assepticamente, após fluxo de 2 minutos, conforme recomendações da American Public Health Association ${ }^{2}$, em intervalos semanais, processadas em duplicata e transportadas em caixas isotérmicas ao Laboratório de Pesquisa em Microbiologia Aplicada (LaPeMA) da Universidade de Franca, não ultrapassando duas horas entre a coleta e o início dos procedimentos laboratoriais.

Isolamento e quantificação dos fungos filamentosos. As amostras foram processadas, em capela de fluxo laminar, empregando-se a técnica da membrana filtrante, conforme descrito anteriormente ${ }^{2}$, utilizando-se membranas (Millipore ${ }^{\circledR}$ ) com poros de $0,45 \mu \mathrm{m}$ para procedimento quantitativo visando à concentração da amostra. As membranas foram colocadas sobre ágar Sabouraud-dextrose (SDA) (Difco Labs, Detroit, Mich, USA), suplementado com cloranfenicol $(30 \mathrm{mg} / 100 \mathrm{~mL})$ (Sigma, ST. Louis, MO, USA) para isolamento das colônias fúngicas, mantidas em estufa com demanda de oxigênio (BOD) a $30^{\circ} \mathrm{C}$ por duas a três semanas, sendo examinadas a partir do segundo dia. Das colônias que apresentaram a mesma morfologia, dentro de uma mesma placa foram subcultivadas apenas uma para identificação.

Após a contagem das colônias, os resultados foram expressos $\mathrm{em} \mathrm{UFC} / \mathrm{mL}^{2}$.

Identificação dos fungos filamentosos. A identificação dos fungos filamentosos ao nível de gênero foi realizada com análise morfológica da colônia por técnica de colônia gigante e microscópica, bem como técnica de microcultivo em ágar-batata dextrose $e^{293031}$.

\section{RESULTADOS}

Realizou-se 15 coletas de água nos sete pontos do sistema de distribuição e máquinas, obtendo-se um total de 100 amostras de água que foram constituídas de 89 (89\%) do sistema de distribuição e 11 (11\%) das máquinas de hemodiálise.

Foram recuperados, no total, 116 isolados de fungos filamentosos. A partir do abastecimento público, foram recuperados 21 isolados com prevalência de Aspergillus sp e Cladosporium sp. Estes resultados estão descritos na Tabela 1.

Das amostras coletadas a partir dos pontos de pós-tratamento (osmose reversa) e do reservatório de armazenamento, houve prevalência de Cladosporium sp (11 isolados) e Trichoderma sp (10 isolados). Estes dois pontos apresentaram praticamente 0 mesmo número de isolados, sendo que, os gêneros Cladosporium

Tabela 1 - Número de isolados fúngicos obtidos a partir de 15 coletas do sistema público de distribuição de água, de uma Unidade de hemodiálise do interior do Estado de São Paulo.

\begin{tabular}{lc}
\hline Fungos isolados & Isolados $\left(\mathrm{n}^{\circ}\right)$ \\
\hline Aspergillus $\mathrm{sp}$ & 7 \\
Cladosporium $\mathrm{sp}$ & 6 \\
Micelia sterilia & 4 \\
Exophiala $\mathrm{sp}$ & 1 \\
Fonsecaea $\mathrm{sp}$ & 1 \\
Streptomyces $\mathrm{sp}$ & 1 \\
Trichoderma $\mathrm{sp}$ & 1 \\
\hline Total & $\mathbf{2 1}$ \\
\hline
\end{tabular}

$\mathrm{n}^{0}=$ número total de isolados obtidos. 
e Trichoderma foram encontrados em ambos os pontos. A relação completa dos isolados fúngicos nestes pontos está descrita na Tabela 2 .

Nas salas onde se realizam as sessões de diálise foram recuperados 49 isolados de fungos filamentosos. Fusarium sp, Cladosporium sp e Trichoderma sp foram isolados nas três salas (Tabela 3), com predominância desse último fungo com igual número de isolados em todas as coletas (Tabela 3).

Tabela 2 - Número de isolados fúngicos recuperados a partir de 15 amostras nos pontos de pós-osmose e reservatório de estocagem da água tratada, de uma Unidade de hemodiálise do interior do Estado de São Paulo.

\begin{tabular}{lcc}
\hline Microrganismo & Pós-osmose $\left(\mathrm{n}^{\mathrm{o}}\right)$ & Tanque reservatório $\left(\mathrm{n}^{\mathrm{0}}\right)$ \\
\hline Cladosporium $\mathrm{sp}$ & 11 & 2 \\
Trichoderma $\mathrm{sp}$ & 2 & 10 \\
Aspergillus $\mathrm{sp}$ & 4 & 0 \\
Fusarium $\mathrm{sp}$ & 0 & 2 \\
Bipolaris $\mathrm{sp}$ & 1 & 0 \\
Streptomyces $\mathrm{sp}$ & 1 & 0 \\
Acremonium $\mathrm{sp}$ & 0 & 1 \\
Penicillium $\mathrm{sp}$ & 0 & 1 \\
Micelia sterilia & 0 & 1 \\
\hline Total & 19 & 17 \\
\hline
\end{tabular}

$\mathrm{n}^{0}=$ número total de isolados obtidos.

Tabela 3 - Distribuição dos 49 isolados fúngicos nas três salas de diálise em 15 coletas estudadas, em uma Unidade de hemodiálise do interior do Estado de São Paulo.

\begin{tabular}{lccc}
\hline Microrganismo & Sala A $\left(\mathrm{n}^{\mathrm{o}}\right)$ & Sala B $\left(\mathrm{n}^{\mathrm{o}}\right)$ & Sala C $\left(\mathrm{n}^{\mathrm{o}}\right)$ \\
\hline Trichoderma $\mathrm{sp}$ & 10 & 10 & 10 \\
Cladosporium $\mathrm{sp}$ & 1 & 4 & 3 \\
Fusarium $\mathrm{sp}$ & 3 & 1 & 4 \\
Penicillium $\mathrm{sp}$ & 1 & 0 & 0 \\
Aspergillus $\mathrm{sp}$ & 1 & 0 & 1 \\
\hline Total & 16 & 15 & 18 \\
\hline
\end{tabular}

$\mathrm{n}^{\mathrm{o}}=$ número total de isolados obtidos.

Os fungos recuperados a partir das máquinas de hemodiálise totalizaram 10 isolados também com predominância do gênero Trichoderma (4), seguido por Aspergillus (3), Cladosporium (2) e Fusarium (1).

O número de unidades formadoras de colônias foi calculado como previamente descrito ${ }^{2}$, sendo que todos os valores variaram de 1 a $7 \mathrm{UFC} / \mathrm{mL}$ nos diferentes pontos de coleta. Os valores encontrados, descritos por ponto, assim como a média das contagens estão descritos na Tabela 4. As maiores contagens referiram-se à rede

Tabela 4 - Intervalos e médias dos valores encontrados em UFC/mL por ponto de coleta, em 100 amostras de água de uma Unidade de bemodiálise do interior do Estado de São Paulo.

\begin{tabular}{lcr}
\hline Ponto de coleta & Intervalo encontrado & Média \\
\hline Rede pública & $1-7$ & 4 \\
Pós-osmose & $2-5$ & 2,8 \\
Tanque reservatório & $2-6$ & 3,9 \\
Sala A & $1-4$ & 2,1 \\
Sala B & $1-3$ & 1,4 \\
Sala C & $1-6$ & 1,8 \\
Máquinas & $1-3$ & 1,3 \\
\hline
\end{tabular}

pública (média $=4 \mathrm{UFC} / \mathrm{mL}$ ), embora o tanque reservatório tenha apresentado número praticamente igual (média = 3,9UFC/mL).

\section{DISCUSSÃo}

É bem conhecido que a qualidade dos fluídos de diálise dependem de uma complexa cadeia de dispositivos e procedimentos, os quais incluem o monitoramento do sistema hídrico e o controle de qualidade dos procedimentos implantados. Reações pirogênicas e sépsis são consequiências potenciais da deficiência na qualidade da água tratada utilizada para a preparação do dialisado. Vários microrganismos podemse multiplicar rapidamente em variedades de fluidos nas unidades de hemodiálises, incluindo destilação, deionização, osmose reversa e água do suavizador, que são normalmente consideradas desprovidas de nutrientes ${ }^{48}$. A formação de biofilmes, que facilitam a persistência microbiana nos diferentes pontos do sistema e protegem o microrganismo da desinfecção, aumentam o risco de contaminação e elevam os níveis de endotoxinas na água ${ }^{35}$.

A incidência de infecções fúngicas oportunísticas causadas por fungos filamentosos no ambiente hospitalar, tem aumentado, apesar da implementação na metodologia diagnóstica e terapêutica, sugerindo a presença de fontes de manutenção e propagação desses fungos, não sendo clinicamente relevante à presença destes no ambiente aquático ${ }^{73637}$. Além disso, vários estudos recentes ${ }^{375}$ têm relatado o encontro de fungos patogênicos em águas a despeito dos padrões adequados de cloração da água ( $0,3 \mathrm{ppm}$ de cloro). Assim, devido à inexistência de padronização qualitativa ou quantitativa para fungos, em nível nacional ou internacional, investigou - se a presença destes microrganismos no sistema de distribuição de água de uma unidade de hemodiálise.

Existem poucos relatos na literatura com respeito à ocorrência de fungos em águas tratadas e amostras de dialisado. Em nosso estudo obtivemos 116 isolados de fungos filamentosos a partir de 100 amostras de água (1L). Recuperamos fungos filamentosos em $100 \%$ das amostras. Bambauer e cols ${ }^{9}$ recuperaram fungos filamentosos em 10\% das amostras de água tratadas e em $20 \%$ das amostras de água do dialisado quando analisou 30 Centros de hemodiálise, na Alemanha. Contudo, esses autores, não usaram meios específicos e não incubaram por mais de 48 horas, as placas. Além disso, inocularam $0,1 \mathrm{~mL}$ de cada amostra usando a técnica spread plate. Em nosso estudo, além de usarmos volume muito superior (1L) aplicamos a metodologia da membrana filtrante que concentra o volume. A incubação por duas a três semanas, a $30^{\circ} \mathrm{C}$ em meio universal para fungos (ágar Sabouraud) pode ter contribuído para nossa grande positividade nas culturas.

Dos 116 fungos os mais comumente isolados neste estudo, foram 47 (40,5\%) Trichoderma sp, 29 (25\%) Cladosporium sp, $16(13,8 \%)$ Aspergillus sp e 11 (9,5\%) Fusarium sp. Nos poucos estudos semelhantes anteriormente relatados ${ }^{4}{ }^{24}$ não há homogeneidade na predominância do gênero isolado, sendo Penicillium sp, fungos demáceos (Cladosporium sp) e Aspergillus fumigatus os fungos isolados por estes autores. 
Trichoderma sp (47/116 - 40,5\%) foi o mais freqüente fungo filamentoso isolado. Trichoderma sp é amplamente distribuído no solo e vegetais. As infecções por Trichoderma têm caráter oportunista e desenvolvem-se em pacientes imunocomprometidos tais como neutropênicos, transplantados, pacientes renais crônicos e pacientes com doenças crônicas pulmonares ${ }^{2349} .0$ isolamento de Trichoderma (Trichoderma koningii) em águas é relatado por Sage e cols ${ }^{43}$, onde é associado à poluição, em oposição a este trabalho.

Aspergillus sp foi prevalente na água afluente (7/21) em nosso estudo e, também, encontrado na água pós-tratamento onde totalizou $21 \%$ (4/19) dos isolados. No circuito aquático das salas onde se realizam as diálises, foi recuperado em apenas 4\% (2/49), embora das máquinas de diálise seu nível de recuperação tenha sido de 30\% (3/10). 0 gênero Aspergillus é constituído por 185 espécies ${ }^{32}$, sendo os principais patógenos humanos Aspergillus fumigatus, Aspergillus flavus e Aspergillus niger e, a principal patologia relacionada a pacientes imunodeficientes, a aspergilose invasiva $^{28}{ }^{29}$. Embora esteja descrito que a aspergilose é primariamente uma infecção aérea, adquirida por inalaçã̃o ${ }^{47}$, vários estudos têm sugerido que a água seja possível fonte de infecção por estes fungos ${ }^{471118}$. Adicionalmente, é possível que a presença de Aspergillus no ambiente aquático potencialize a colonização devido a sua grande capacidade de esporulação em pequeno período de tempo, formando micronichos ambientais que não são afetados pelos procedimentos regulares de desinfecção ${ }^{417}$. Em estudo prévio ${ }^{7}$, Aspergillus sp foi também o fungo encontrado prevalentemente tanto na água tratada como nas amostras do dialisado.

Tem sido sugerido que a diferença de temperatura encontrada entre os diversos segmentos que compõem o circuito hídrico influencia 0 crescimento microbiano ${ }^{51}$, sendo favorecido em temperaturas mais elevadas. Assim, a elevação da temperatura nas máquinas de diálise $\left(37^{\circ} \mathrm{C}\right)$ e no sistema de osmose reversa hipoteticamente pode favorecer o crescimento do fungo, pois estudo anterior ${ }^{14}$ associa aumento do crescimento de Aspergillus à umidade $\mathrm{e}$ aumento de temperatura.

Penicillium sp são também conhecidos produtores de micotoxinas e têm sido isolados em suplementos de água de rede pública em Portugal ${ }^{21}$, de hospitais na Grécia e Alemanha ${ }^{22}$.

Em nosso estudo, Fusarium sp foi recuperado a partir do tanque reservatório de água tratada (pós-osmose) e, daí por diante, em todo o circuito e máquinas da unidade de hemodiálise em porcentagem variada $(6,7 \%$ - sala B a $22,2 \%$ - sala C). A capacidade de formação de biofilmes (estruturas microbianas comunitárias aderidas a superfícies e envolvidas por matriz de material exopolimérico que servem de reservatório ou fonte contínua de microrganismos viáveis ${ }^{12}$ ) por espécies de Fusarium foi demonstrada por Honraet e cols ${ }^{25}$. Entretanto, é bem conhecido que espécies de Fusarium geralmente não causam doenças sistêmicas em pacientes imunocompetentes, contudo, são produtores notáveis de várias micotoxinas carcinogênicas e imunossupressivas ${ }^{1939} 46$. Paralelamente, têm sido descrito que pacientes que abrigam membros do complexo Fusarium solani, sob terapia imunossupressivas, podem tornar-se devastadoramente

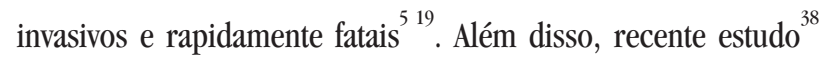
concluiu, após comparação filogenética, entre isolados de Fusarium provenientes de espécimes clínicos e isolados ambientais, inclusive isolados aquáticos hospitalares, que a patogenia de Fusarium (Fusarium solani) se devia à adaptação destas espécies ambientaisà pacientes susceptíveis, principalmente se imunocomprometidos.

No Brasil, Nucci e cols ${ }^{37}$ relataram o encontro de Exopbiala jeanselmei (3,5\%) como fungo contaminante de solução antisséptica utilizada em um hospital, os autores sugeriram ser a água utilizada para a preparação dessa solução a fonte desses microrganismos. Neste estudo recuperou-se um isolado (0,9\%) de Exophiala sp confirmando a presença desse fungo em ambiente aquático hospitalar, onde estão expostos pacientes de alto risco. Os resultados desses estudos permitem-nos inferir que a água do ambiente hospitalar seja fonte potencial de transmissão desses fungos, gerando perspectivas no desenvolvimento de medidas de controle no intuito de minimizar a exposição dos pacientes imunocomprometidos. Estudos epidemiológicos baseados na caracterização molecular e comparação dos isolados fúngicos recuperados de pacientes e do ambiente são necessários, no futuro, para expandir o entendimento dessas rotas alternativas de transmissão.

Uma das limitações deste estudo foi a identificação apenas em nível de gênero dos fungos isolados, justificado pela pouca acurácia das técnicas convencionais, principalmente para isolados ambientais. Os métodos moleculares corroboram com o processo de identificação, mas ainda é um recurso dispendioso e que necessita de pessoal altamente especializado e que não está disponível para a maioria dos laboratórios.

Em estudos anteriores ${ }^{727}$ foram documentados que a água purificada pós-osmose reversa aumentou significativamente a contagem de fungos filamentosos em comparação com a água afluente ao sistema. Opostamente, em nosso estudo, houve uma diminuição de praticamente $25 \%$ nas contagens da água purificada (4 para 2,8UFC/mL), retornando praticamente aos níveis iniciais no tanque reservatório $(3,9 \mathrm{UFC} / \mathrm{mL})$ e novamente diminuindo no circuito. 0 aumento e posterior diminuição no tanque e circuito, respectivamente, poderia ser explicado pela diminuição do fluxo no reservatório e aumento deste no circuito, uma vez que estudos prévios ${ }^{264145}$ comprovam a eficácia do fluxo contínuo e a periculosidade da estagnação da água.

Assim, o monitoramento e a manutenção dos equipamentos de purificação da água são cruciais para a qualidade microbiana e química da água produzida. A padronização estabelecida pelos órgãos competentes nacionais e internacionais visa à eliminação de vários componentes químicos, bacterianos e endotoxinas, embora o encontro de fungos ainda não tenha conseguido a atenção necessária das agências regulatórias. Sendo os resultados desta pesquisa coincidentes com os obtidos em estudos internacionais, sugerimos que os suprimentos de água em unidades de hemodiálise devam ser monitorados também quanto à presença de fungos filamentosos, pois face ao exposto, os fungos encontrados em nossa pesquisa são considerados potencialmente patogênicos, sendo necessária a adoção de medidas profiláticas eficazes, minimizando a exposição destes pacientes imunodeficientes à fontes aquáticas ambientais contaminadas. 


\section{REFERÊNCIAS}

1. Abbott KC, Hypolite I, Tveit D, Hshieh P, Cruess D, Agodoa LY. Hospitalizations for fungal infections after initiation of chronic dialysis in the United States. Nephron 89: 426-432, 2001

2. American Public Health Association (APHA). Standard Methods for the Examination of Water and Wastewater p. 9-33,1998.

3. Anaissie EJ, Kuchar RT, Rex JH, Francesconi A, Kasai M, Makaller FMC, LozanoChiu M, Summerbell RC, Dignani MC, Chanock SJ, Walsh TJ. Fusariosis associated with pathogenic Fusarium species colonization of a hospital water system: a new paradigm for the epidemiology of opportunistic mold infections. Clinical Infections Diseases 33:1871-1878, 2001.

4. Anaissie EJ, Stratton Sl, Dignani MC, Summerbell RC, Rex JH, Monson TP, Spencer T, Kasai M, Francesconi A, Walsh TJ. Pathogenic Aspergillus species recovered from a hospital water system: a 3-year prospective study. Clinical Infections Diseases 34:780-789, 2002.

5. Arrese JE, Piérard-Franchimont C, Piérard GE. Fatal hyalohyphomycosis following Fusarium onychomycosis in an immunocompromised patient. The American Journal of Dermatopathology 18:196-198, 1996.

6. Arvanitidou M, Spaia S, Katsinas C, Pangidis P, Constantinidis T, Katsouyannopoulos $\mathrm{V}$, Vayonas G. Microbiological quality of water and dialysate in all haemodialysis centres of Greece. Nephrology Dialysis Transplantation 13:949-954, 1998.

7. Arvanitidou M, Spaia S, Velegraki A, Pazarloglou M, Kanetidis D, Pangidis P, Askepidis N, Katsinas CH, Vayonas G, Katsouyannopoulos V. High level of recovery of fungi from water and dialysate in haemodialysis units. The Journal Hospital Infection 45:225-230, 2000.

8. Association for the Advacement of Medical Instrumentation (AAMI). Standards and recommended practices. Dialysis 3: 293-300, 1993

9. Bambauer R, Schauer M, Jung WK, Daum V, Vienken J. Contamination of dialysis water and dialysate. A Survey of 30 Centers. Asayo Journal 40: 1012-1016, 1994.

10. Boulton-Jones IM, Vick R, Cameron J, Black D. Immune responses in uremia. Clinical Nephrology 1:350-360, 1973.

11. Chazalet V, Debeaupuis JP, Sarfatij. Molecular typing of environmental and patients isolates of Aspergillus fumigatus from various hospital settings. Journal Clinical Microbiology 36:1494-1500, 1998.

12. Davey MEE, O'Toole G. Microbial Biofilms: From ecology to molecular genetics. Microbiology and Molecular Biology Reviews 64:847-867, 2000.

13. Denning DW. Aspergillus Species. In: Mandell GL, Bennett JE, Dolin R eds. Principles and Pratice of Infectious Diseases. $5^{\text {th }}$ edition, volume 2. Churchill, Livingstone, Philadelphia, PA, p. 2674-2684, 2000.

14. Esteban A, Abarca ML, Bragulat MR, Cabanes FJ. Study of the effect of water activity and temperature on ochratoxin a production by Aspergillus carbonarius. Food Microbiology 23:634-640, 2006.

15. European Best Practice Guidelines for Haemodialysis (EBPG) Part 1. Dialysis fluid purity. Journal de Physique. Nephrology Dialysis Transplantation 17:45-62, 2002.

16. Favero MS, Carson LA, Bond WW, Petersen NJ. Factors thet influence microbial contamination of fluids associated with hemodialysis machines. Applied Microbiology 28:822-830, 1975.

17. Gercovich FG, Richman SP, Rodriguez V, Luna M, Mccardie KB, Bodey GP. Successful control of systemic Aspergillus niger infection in two patients with acute leukemia. Cancer 36: 2271-2276, 1975.

18. Girardin H, Sarfati J, Traore F, Dupouy CJ, Derouin F, Latge JP. Molecular epidemiology of nosocomial invasive aspergillosis. Journal Clinical Microbiology 32:684-690, 1994.

19. Girmenia C, Arcese W, Micozzi A, Martino P, Bianco P, Morace G. Onychomycosis as a possible origin of disseminated Fusarium solani infection in a patient with severe aplastic anemia. Clinical Infections Diseases 14:1167, 1992.

20. Goldblum SE, Reed WP. Host defences and immunological alterations associated with chronic hemodialysis. Annales de Droit International Médical 93: 597-613, 1980.

21. Gonçalves AB, Paterson RR, Lima R. Survey and significance of filamentous fungi from tap water. International Journal of Hygiene and Environmental Health 209:257-264, 2006
22. Gottlich E, van der Lubbe W, Lange B, Fiedler S, Melchert I, Refenrath M, Flemming HC, Hoog S. Fungal flora in groundwater - derived public drinking water. International Journal of Hvgiene and Environmental Health 205:269-279, 2002

23. Groll AH, Walsh TJ. Uncommon opportunistic fungi: new nosocomial threats. Clinical Microbiology and Infection 7:8-24, 2002.

24. Hinzelin F, Block JC. Yeast and filamentous fungi. in drinking water. Environmental Technology Letters 6:101-105, 1985.

25. Honraet K, De Vos MM, Summerbell RC, Van Kempen I, De Saeger S, Vermeersch H, Van Peteghem C, Nelis HJ. Recurrent colonization of sucessively implanted tracheoesophageal vocal prostheses by a member of the Fusarium solan $i$ species complex. Journal of Clinical Microbiology 43:770-777, 2005.

26. Jaber BL. Bacterial infections in hemodialysis patients: pathogenesis and prevention. Kidney International 67:2508-2519, 2005.

27. Klein E, Pass T, Harding GB, Wright R, Million C. Microbial and endotoxin contamination in water and dialysate in central United States. Artificial Organs 14:85-94, 1990.

28. Kwon-Chung KJ, Bennett JE. Medical Mycology. Lea \& Febiger, Philadelphia, 1992.

29. Lacaz CS, Porto E, Heins-Vaccari EM, Melo NT. Guia para identificação de fungos, actinomicetos e algas de interesse médico. Editora Sarvier, São Paulo, 1998.

30. Larone D. Medically Important Fungi. $3^{\mathrm{a}}$ edition. Asm Press, Washington DC, 1995.

31. Mendes-Giannini MJ, Melhem MSC. Fungos. In: Ferreira WE, Souza S, Ávila SLM (coords) Diagnóstico das principais doenças infecciosas e Autoimunes. $2^{\mathrm{a}}$.edição, Editora Guanabara-Koogan, Rio de Janeiro, 2002.

32. Michael JK, Sigler L. In: Murray PR, Baron EJO, Pfaller MA, Tenover FC, Yolken RH (eds) Manual of Clinical Microbiology 97:1212-1235, 1999.

33. Ministério da Saúde Portaria $\mathrm{n}^{\circ} 154$ de 15.06.2004. D.0.U. 17.06.2004 Regulamento técnico para o funcionamento dos serviços de diálise. Brasil, 2004

34. Modai D, Berman S, Sheley Y, Cohn M, Weissgarten J, Averbukh Z. Interleukin -2 receptor in similarly expressed by activated lymphocytes from patients on chronic hemodialysis and healthy subjects. Clinical Immunology and Immunopathology 55:237-241, 1990 .

35. Morin P. Identification of the bacteriological contamination of a water treatment line used for hemodialysis and its disinfection. The Journal Hospital Infection 45:218-224, 2000

36. Nagy LA, Olson BH. The ocorrence of filamentous fungi in water distribuion systems. Journal of Microbiology 28:667-671, 1983 .

37. Nucci M, Akiti T, Barreiros G, Silveira F, Revankar SG, Wickes BL, Patterson TF. Nosocomial outbreak of Exophiala jeanselmei fungemia associated with contamination of hospital water. Clinical Infections Diseases 34:1475-1480, 2002

38. O'Donnell K. Molecular phylogeny of the Nectria baematococca Fusarium solani species complex. Mycología 92:919-938, 2000.

39. O'Donnell K, Sutton DA, Rinaldi MG, Magnon KC, Cox PA, Revankar SG, Sanche S, Geiser DM, Juba JH, van Burik JA, Padhye A, Anaissie EJ, Francesconi A, Walsh TJ, Robinson JS. Genetic diversity of human pathogenic members of the Fusarium oxysporum complex inferred from multilocus DNA sequence data and amplified fragment length polymorphism analyses: evidence for the recent dispersion of a geographically wides pread clonal lineage and nosocomial origin. Journal of Clinical Microbiology 42:5109-5120, 2004.

40. Pizzarelli F, Cerrai T, Biagini M, Malaguti M, Bargagna R. Dialysis water treatment systems and monitoring in Italy: Reults of a national survey. Journal Nephrology 17: 565-569, 2004.

41. Powe NR, Jaar B, Furth SL, Hermann J, Briggs W. Septicemia in dialysis patients: incidence, risk factors, and prognosis. Kidney International 55:1081-1090, 1999.

42. Rosa CM. Hemodiálise e risco biológico. http://www.riscobiologico.org/risco// hemo.htm\#>. Acesso em 19 outubro, 2006.

43. Sage AE, Vasil AI, Vasil ML. Molecular characterization of mutants affected in the osmoprotectant-dependent induction of phospholipase C in Pseudomonas aeruginosa PA01. Molecular Microbiology 23:43-56, 1997. 
44. Silva AM, Martins CTB, Ferraboli R, Jorgetti V, Junior JER. Revisão/Atualização em diálise: água para hemodiálise. Jornal Brasileiro de Nefrologia 18:180-188, 1996.

45. Sugiura Y, Barr JR, Barr DB, Brock JW, Elie CM, Ueno Y, Patterson DG, Potter ME, Reiss. E Physiological characteristics and mycotoxins of human clinical isolates of Fusarium species. Mycological Research 103:1462-1468, 1999.

46. Vanderbergh MF, Verwei JPE, Voss A. Epidemiology of nosocomial fungal infections: invasive aspergillosis and the environment. Diagnostic Microbiology and Infections Disease 34:221-227, 1999.

47. Vorbeck-Meister I, Sommer R, Vorbeck F, Hörl WH. Quality of water used for haemodilysis: bacteriological and chemical parameters. Nephrology Dialysis Transplantation 14:666-675, 1999.

48. Walsh TJ, Dixon DM. Nosocomial aspergillosis, environmental microbiology, hospital epidemiology, diagnosis and treatment. European Journal of Epidemiology 5:131-142, 1989 .
49. Warris A, Gaustad P, Meis JFGM, Voss A, Verweij PE, Abrahamsen TG. Recovery of filamentous fungi from water in a pediatric bone marrow transplantation unit. The Journal Hospital Infection 47:143-148, 2001.

50. Zhang N, O'Donnell K, Sutton DA, Nalim FA, Summerbell RC, Padhye AA, Geiser DM. Members of the Fusarium solani species complex that cause infections in both humans and plants are common in the environment. Journal of Clinical Microbiology 44:2186-2190, 2006.

51. Zunino P, Beltrán L, Zunino L, Méndez H, Percovich V, Rocca R, Antonelli B. Microbiological quality of hemodialysis water in a three-year multicenter study in Uruguay. Journal of Nephrology 15:374-379, 2002. 